Original Article

\title{
Effects of joint immobilization and hindlimb unloading on collagen fibers of soleus muscles in rats
}

Tomohiro Matsumoto, MS, RPT 1)*, Takeya Ono, PhD, RPT ${ }^{2,3)}$, Hideki Ishikura, PhD, RPT ${ }^{4}$, Kazuki Aihara, MS, RPT ${ }^{3)}$, Yuta Sato, MS, RPT ${ }^{3)}$, Atsushi Tasaka, PhD, RPT ${ }^{5)}$, Namiko Umei, PhD, RPT ${ }^{2)}$, Wakako Tsumiyama, PhD, RPT ${ }^{2)}$, Sadahi Oki, PhD, $\mathrm{MD}^{2,3)}$

1) Department of Rehabilitation Science, Kobe University Graduate School of Health Sciences: 7-10-2 Tomogaoka, Suma-ku Kobe City, Hyogo 654-0142, Japan

2) Department of Physical Therapy, Faculty of Health and Welfare, Prefectural University of Hiroshima, Japan

3) Graduate School of Comprehensive Scientific Research, Prefectural University of Hiroshima, Japan

4) Department of Rehabilitation, Physical Therapist, Faculty of Health Sciences, Hiroshima Cosmopolitan University, Japan

5) Department of Rehabilitation Science, Osaka Health Science University, Japan

\begin{abstract}
Purpose] The purpose of this study was to determine whether collagen fibers cause a difference in a contracture resulting from a combination of joint fixation and hindlimb unloading as compared to joint fixation only. [Subjects and Methods] The subjects of this study were 21 female Wistar rats divided into 4 groups as follows: Control Group (CON, n=7); Joint Fixation Group (JF, n=7), Hindlimb Unloading Group (HU, n=7), Joint Fixation Plus Hindlimb Unloading Group (JF+HU, n=7). This study was conducted for 1-week. Ankle joint range of motion and positive areas of collagen using fluorescent stain were analyzed. [Results] Ankle joint range of motion in JF + HU showed an increase compared to that of JF. Positive areas of Type I collagen in JF+HU showed an increase as compared with that of JF. [Conclusion] The results of this study suggested that the difference in a contracture caused by only joint immobilization and by a combination of joint immobilization and hindlimb unloading were significantly associated with Type I collagen.

Key words: Joint immobilization, Hindlimb unloading, Collagen
\end{abstract}

(This article was submitted Oct. 26, 2016, and was accepted Apr. 23, 2017)

\section{INTRODUCTION}

Contractures, which include the skin, skeletal muscle, tendon, and joint capsule, result in a decrease of joint range of motion $(\mathrm{ROM})^{1,2)}$ and are caused by joint immobilization ${ }^{1-4)}$. It has been reported that skeletal muscles particularly participate in contractures more than other tissues ${ }^{1}$. Collagen fiber, which is the main structural protein in several tissues, is also present in skeletal muscle and is classified by several types of function and structure ${ }^{5)}$ In particular, Type I and Type III collagen fibers are the primary isoforms of skeletal muscle ${ }^{5-7}$. It has been reported that collagen fibers in skeletal muscle increase with joint immobilization, and an increase in collagen fibers causes an increase in the degree of contractures 3,4 ).

Experiments related to contractures are often conducted with animals ${ }^{1-4)}$. To generate contractures, joint fixation using a cast is performed in experiments with animals ${ }^{1-4)}$. In recent years a combination of joint fixation and hindlimb unloading also causes contractures similar to only joint fixation and are thought to be close to bed rest-causes contractures clinically ${ }^{8)}$.

*Corresponding author. Tomohiro Matsumoto (E-mail: tomohiro19900804@gmail.com)

(C2017 The Society of Physical Therapy Science. Published by IPEC Inc.

This is an open-access article distributed under the terms of the Creative Commons Attribution Non-Commercial No Derivatives (by-nc-nd) License. (CC-BY-NC-ND 4.0: https://creativecommons.org/licenses/by-nc-nd/4.0/) 
However it has been found that contractures caused by a combination of joint fixation and hindlimb unloading tend to increase as compared with that of only joint fixation, but the causing factor is not clear. We hypothesized that the factor involves the collagen fibers. Collagen isoforms are thought to affect the mechanical properties of muscle. Type I collagen is generally related to tissue stiffness (i.e., resistance to stretch), whereas the abundance of Type III collagen correlates with tissue compliance (i.e., ability to elongate readily). Therefore, the muscle collagen may be analyzed separately divided into type I and III collagen. The purpose of this study was to determine whether collagen fibers cause a difference in contractures from a combination of joint fixation and hindlimb unloading than from only joint fixation.

\section{SUBJECTS AND METHODS}

Twenty-one female 8-week-old Wistar rats with a body weight of $247.2 \pm 7.7 \mathrm{~g}$ (mean $\pm \mathrm{SD}$ ) were used. The animals were housed in a temperature-controlled room at $23^{\circ} \mathrm{C}$ on a $12 \mathrm{~h}$ light-dark cycle and were given free access to standard rat food and water. This study was performed according to the Regulations on Animal Experiments of the Animal Experiments Committee (Approval Number: 13 MA 006).

All of the rats were divided into 3 groups as follows: Control Group (CON, n=7), Joint Fixation Group (JF, n=7), Hindlimb Unloading Group (HU, n=7), and Joint Fixation Plus Hindlimb Unloading Group (JF+HU, n=7). The same animals were used for the HU (left hindlimb) and JF+HU (right hindlimb). The experimental period was one week. Under pentobarbital sodium $(40 \mathrm{mg} / \mathrm{kg})$, joint fixation was applied to $\mathrm{JF}$ and $\mathrm{JF}+\mathrm{HU}$ by fixing the right ankle joint of rats in full plantar flexion with a non-elastic tape. Moreover, hindlimb unloading was applied to HU and JF+HU by suspending the tails. A $1.0 \mathrm{~mm}$ $\mathrm{K}$-wire was inserted through the tails of JF+HU. The wire was attached to a swivel hook, and then its length was adjusted so that the hindlimb did not touch the cage bottom. The forelimbs were allowed to maintain contact with the floor of cage, with water and food access ad libitum.

ROM of ankle joint dorsiflexion was measured according to Sato et $\mathrm{al}^{8}{ }^{8}$. Briefly, after the rat was anesthetized with pentobarbital sodium $(40 \mathrm{mg} / \mathrm{kg})$, the rat was positioned on its side, and a force of $0.3 \mathrm{~N}$ was applied perpendicularly to the sole of the foot by a tension meter (LTS-1KA; Kyowa Electronic Instruments Co., Ltd., Japan). Then, a digital photograph was taken from directly above the hindlimb, and the angle of dorsiflexion was measured with computer software (image J 1.44p, U.S. National Institutes of Health, Bethesda, MD, USA). To eliminate forefoot movement from the measurement, the dorsiflexion ROM was defined as the angle obtained from a line parallel to the longitudinal axis of the fibula and a line parallel to the bottom of the heel. During ROM measurements, the knee joint was flexed.

The rats were sacrificed while under the pentobarbital sodium after the ROM measurements were done. The soleus muscles of the hindlimb were excised, weighed, and frozen in isopentane in liquid nitrogen. The samples were stored at $-80{ }^{\circ} \mathrm{C}$ until Immunohistochemical analysis tests was done.

The stored tissues were sliced into $10-\mu \mathrm{m}$-thick sections using a cryostat microtome, and then washed three times for 5 min with $0.01 \mathrm{M}$ phosphate buffer in saline (PBS; $\mathrm{pH}$ 7.4). The sections were then incubated overnight at room temperature with the primary antibodies. The primary antibodies, a mouse monoclonal anti-type I collagen (1:200; Santa Cruz Biotechnology, USA) and a rabbit polyclonal anti-type III collagen (1:200; LSL, Japan), were diluted with 0.01 M PBS and 4\% Triton X-100. After being washed three times for 5 min with $0.01 \mathrm{PBS}$, the sections were incubated overnight at room temperature with secondary antibodies. The secondary antibodies, Alexa Flour-conjugated anti-mouse IgG (1:1000; Life Technologies, USA) and Hilyte Flour-conjugated anti-rabbit IgG (1:1000; Anaspec, USA), were diluted with $0.01 \mathrm{M}$ PBS. Then, the sections were washed three times for $5 \mathrm{~min}$ with $0.01 \mathrm{M}$ PBS. The sections were viewed with a fluorescence microscope (BZ-X700, KEYENCE, Japan). Data for positive areas Type I or III collagen in perimysium and endomysium in soleus muscle are expressed normalized to cross sectional area (CSA), Type I or III collagen /muscle CSA $\times 100(\%)$.

Data are expressed as means \pm standard deviation. The one-way analysis of variance (ANOVA) was used for analysis of muscle wet weight, ankle joint dorsiflexion ROM and positive areas of Type I or III collagen. The Scheffe test was used for post-hoc comparison. The analyses were performed using the SPSS version 13.0 (SPSS Inc.). Significance was accepted for values of $\mathrm{p}<0.05$.

\section{RESULTS}

Body weights and relative (to body weight) soleus weights are shown in Table 1. There was no significant difference among the 4 groups in body weight at the beginning of the experiment. Body weight and relative soleus weight at the end of the experiment in JF, HU and JF+HU were significantly smaller than that in CON.

Dorsiflexion ROMs are shown in Table 2. Mean \pm SD values for dorsiflexion ROM were as follows: at the beginning of the experiment, $135.2 \pm 4.4^{\circ}$ in $\mathrm{CON}, 135.3 \pm 2.9^{\circ}$ in JF, $138.6 \pm 5.1^{\circ}$ in $\mathrm{HU}$, and $136.2 \pm 3.5^{\circ}$ in JF+HU; at the end of the experiment, $134.2 \pm 5.8^{\circ}$ in $\mathrm{CON}, 92.2 \pm 8.9^{\circ}$ in $\mathrm{JF}, 142.7 \pm 2.5^{\circ}$ in $\mathrm{HU}$, and $63.5 \pm 8.9^{\circ}$ in $\mathrm{JF}+\mathrm{HU}$. No significant difference was observed among the 4 groups in dorsiflexion ROM at the beginning of the experiment. Dorsiflexion ROM of JF and $\mathrm{JF}+\mathrm{HU}$ at end of the experiment significantly decreased compared with CON and HU. Moreover, dorsiflexion ROM in $\mathrm{JF}+\mathrm{HU}$ was significantly smaller than that in JF.

Relative data for the positive areas of Type I and III collagen in soleus muscles are shown in Table 2. Mean \pm SD values for 
Table 1. Body weight and muscle mass

\begin{tabular}{lccc}
\hline & $\begin{array}{c}\text { Body mass } \\
(\mathrm{g})\end{array}$ & $\begin{array}{c}\text { Muscle mass } \\
(\mathrm{mg})\end{array}$ & $\begin{array}{c}\text { Relative muscle-to-body mass } \\
(\mathrm{mg} / 100 \mathrm{~g})\end{array}$ \\
\hline CON & $254 \pm 11$ & $140.1 \pm 9.4$ & $55.3 \pm 4.7$ \\
$\mathrm{JF}$ & $242 \pm 11$ & $106.8 \pm 14.7^{*}$ & $44.0 \pm 4.9^{*}$ \\
HU & $239 \pm 7^{*}$ & $97.9 \pm 9.0^{*}$ & $41.1 \pm 3.4^{*}$ \\
$\mathrm{JF}+\mathrm{HU}$ & $239 \pm 7^{*}$ & $91.8 \pm 8.4^{*}$ & $38.5 \pm 3.0^{*}$ \\
\hline
\end{tabular}

Values are means $\pm \mathrm{SD}$.

${ }^{*} \mathrm{p}<0.05$, significant difference compared to CON

Table 2. ROM and positive area of type I and III collagen

\begin{tabular}{lccc}
\hline & ROM & Type I collagen (\%) & Type III collagen (\%) \\
\hline CON & $134.2 \pm 5.8$ & $5.8 \pm 0.8$ & $36.4 \pm 1.4$ \\
JF & $92.2 \pm 8.9^{*}$ & $9.7 \pm 1.7^{*}$ & $43.3 \pm 3.1^{*}$ \\
HU & $142.8 \pm 2.7 \dagger$ & $4.3 \pm 1.1 \dagger$ & $40.0 \pm 3.0$ \\
JF+HU & $63.5 \pm 8.9^{*} \dagger+$ & $12.1 \pm 1.1^{*} \dagger+$ & $42.9 \pm 4.9^{*}$ \\
\hline
\end{tabular}

Values are means \pm SD.

$* p<0.05$, significant difference compared to $\mathrm{CON}$

$\dagger p<0.05$, significant difference compared to JF

$\ddagger \mathrm{p}<0.05$, significant difference compared to $\mathrm{HU}$

relative Type I collagen were as follows: $5.79 \pm 0.79 \%$ in CON, $9.65 \pm 1.74 \%$ in JF, $4.29 \pm 1.05 \%$ in HU, and $12.12 \pm 1.06 \%$ in JF+HU; relative Type III collagen were as follows: $36.35 \pm 1.40 \%$ in CON, $43.27 \pm 3.09 \%$ in JF, $39.95 \pm 2.97 \%$ in $\mathrm{HU}$, and $42.90 \pm 4.92 \%$ in JF+HU. Relative Type I and III collagens resulted in a significant increase in JF and JF+HU compared to $\mathrm{CON}$ and HU. Furthermore, relative Type I collagen in JF+HU was smaller than that in JF. However, no significant difference was observed between JF and JF+HU with regards to relative Type III collagen.

\section{DISCUSSION}

Clinically, prevention and improvement for patients with a disuse syndrome are important. Contractures, which are one condition that physical therapists often encounter with a disuse syndrome, are induced by joint immobilization ${ }^{1-4)}$. Sato et al. ${ }^{8)}$ has reported that the degree of contracture was more pronounced in a combination of joint immobilization and hindlimb unloading as compared with only joint immobilization. Since the results of this previous study showed that contractures in $\mathrm{JF}+\mathrm{HU}$ were more pronounced as compared with that in JF, the methods of this study may be considered reliable.

The effects of hindlimb unloading combined with joint immobilization on Type I and III collagens in rat soleus muscles were determined in this study. In the previous study, it had been reported that an over expression of Type I and III collagen was induced by joint immobilization for a week ${ }^{4}$. In this study, with regard to the effects of immobilization on collagen synthesis, the results seen in the previous study ${ }^{4}$ were also observed. However, no significant difference was observed in type III collagen between JF and JF+HU. On the other hand, Type I collagen in JF+HU was higher than that in JF. A muscle contracture from immobilization was prevented by muscle activity induced with muscular electrical stimulation during immobilization ${ }^{9}$. However, in previous study, it was reported that hindlimb unloading decrease muscle activity ${ }^{10)}$. Therefore, it was assumed that muscle activity was lower in JF+HU than in JF, and Type I collagen was promoted more in JF+HU than JF.

Collagen isoforms are thought to affect the mechanical properties of muscle. Type I collagen is generally related to tissue stiffness (i.e., resistance to stretch), whereas the abundance of Type III collagen correlates with tissue compliance (i.e., ability to elongate readily $)^{5,6)}$. In previous study, it was reported that as the immobilization period increased, the contractures gradually became more pronounced ${ }^{11)}$, and Type I collagen increased up until 12 weeks ${ }^{4}$. However, Type III collagen did not change after the 2 weeks of immobilization ${ }^{4}$. In addition, Type III collagen increased with the muscle injury significantly but did not contribute to tensile strength ${ }^{12}$. In this study, the increase of Type I collagen and contracture were significantly more pronounced in JF+HU than in JF, whereas there was no significant difference between JF and JF+HU in Type III collagen. Therefore, compared to Type I collagen, the contribution of Type III collagen to a contracture was small.

With an elongation of a joint immobilization period, contractures gradually progress ${ }^{1,4)}$. Therefore, when studying contractures, prolonged studies are important. However, the length of this study was only one week. We suggest that a more prolonged study is necessary in the future. This study shows that the difference between a contracture caused by only joint immobilization and by a combination of joint immobilization and hindlimb unloading were significantly associated with Type I collagen. This new finding that our study provides is an advancement on the basic information for contractures. In addition, weight bearing on a lower extremity is important for preventing the progression of a contracture. 


\section{REFERENCES}

1) Trudel G, Uhthoff HK: Contractures secondary to immobility: is the restriction articular or muscular? An experimental longitudinal study in the rat knee. Arch Phys Med Rehabil, 2000, 81: 6-13. [Medline] [CrossRef]

2) Tasaka A, Ono T, Oki S, et al.: Study on changes in skin extensibility during the development of joint contracture due to joint immobilization in rats. J Phys Ther Sci, 2015, 27: 2683-2685. [Medline] [CrossRef]

3) Hibino I, Okita M, Inoue T, et al.: Effect of immobilization on insoluble collagen concentration and type I and type III collagen isoforms of rat soleus muscle. J Jpn Phys Ther Assoc, 2008, 11: 1-6. [Medline] [CrossRef]

4) Honda Y, Sakamoto J, Nakano J, et al.: Upregulation of interleukin-1 $\beta /$ transforming growth factor- $\beta 1$ and hypoxia relate to molecular mechanisms underlying immobilization-induced muscle contracture. Muscle Nerve, 2015, 52: 419-427. [Medline] [CrossRef]

5) Burgeson RE: The collagens of skin. Curr Probl Dermatol, 1987, 17: 61-75. [Medline] [CrossRef]

6) Miller TA, Lesniewski LA, Muller-Delp JM, et al.: Hindlimb unloading induces a collagen isoform shift in the soleus muscle of the rat. Am J Physiol Regul Integr Comp Physiol, 2001, 281: R1710-R1717. [Medline]

7) Mays PK, Bishop JE, Laurent GJ: Age-related changes in the proportion of types I and III collagen. Mech Ageing Dev, 1988, 45: 203-212. [Medline] [CrossRef]

8) Sato Y, Ono T, Ishikura H, et al.: Effect of unweighting the hind limbs of rats on joint contractures. Rigakuryoho Kagaku, 2015, 30: 409-412 (in Japanese) [CrossRef]

9) Oki S, Shibata T, Okumura H, et al.: The effects of electric stimulation on the immobilized muscle. Can electrical stimulation prevent muscle contracture? J Phys Med, 1997, 8: 53-57 (in Japanese).

10) Guillot C, Steinberg JG, Delliaux S, et al.: Physiological, histological and biochemical properties of rat skeletal muscles in response to hindlimb suspension. J Electromyogr Kinesiol, 2008, 18: 276-283. [Medline] [CrossRef]

11) Okita M, Yoshimura T, Nakano J, et al.: Effects of reduced joint mobility on sarcomere length, collagen fibril arrangement in the endomysium, and hyaluronan in rat soleus muscle. J Muscle Res Cell Motil, 2004, 25: 159-166. [Medline] [CrossRef]

12) Hurme T, Kalimo H, Sandberg M, et al.: Localization of type I and III collagen and fibronectin production in injured gastrocnemius muscle. Lab Invest, 1991, 64: 76-84. [Medline] 